\title{
BMJ Open Evolving rifampicin and isoniazid mono-resistance in a high multidrug- resistant and extensively drug-resistant tuberculosis region: a retrospective data analysis
}

\author{
Nomonde Ritta Mvelase (D) , ${ }^{1,2}$ Yusentha Balakrishna, ${ }^{3}$ Keeren Lutchminarain, ${ }^{1,2}$ \\ Koleka Mlisana ${ }^{1,2,4}$
}

To cite: Mvelase NR,

Balakrishna Y, Lutchminarain $\mathrm{K}$, et al. Evolving rifampicin and isoniazid mono-resistance in a high multidrugresistant and extensively drug-resistant tuberculosis region: a retrospective data analysis. BMJ Open 2019;9:e031663. doi:10.1136/ bmjopen-2019-031663

- Prepublication history for this paper is available online. To view these files please visit the journal online (http://dx.doi org/10.1136/bmjopen-2019031663).

Received 14 May 2019 Revised 08 October 2019 Accepted 11 October 2019

Check for updates

(C) Author(s) (or their employer(s)) 2019. Re-use permitted under CC BY-NC. No commercial re-use. See rights and permissions. Published by BMJ.

${ }^{1}$ Medical Microbiology, National Health Laboratory Service,

Durban, South Africa

${ }^{2}$ Medical Microbiology, University of KwaZulu-Natal,

Durban, South Africa

${ }^{3}$ Biostatistics Unit, South African Medical Research Council, Durban, South Africa

${ }^{4}$ Centre for the AIDS Programme of Research in South Africa (CAPRISA), Durban, South Africa

Correspondence to Dr Nomonde Ritta Mvelase; dlaminin15@ukzn.ac.za

\section{ABSTRACT}

Objectives South Africa ranks among the highest drugresistant tuberculosis (DR-TB) burdened countries in the world. This study assessed the changes in resistance levels in culture confirmed Mycobacterium tuberculosis (MTB) in the highest burdened province of South Africa during a period where major changes in diagnostic algorithm were implemented.

Setting This study was conducted at the central academic laboratory of the KwaZulu-Natal province of South Africa.

Participants We analysed data for all MTB cultures performed in the KwaZulu-Natal province between 2011 and 2014. The data were collected from the laboratory information system.

Results Out of 88559 drug susceptibility results analysed, $18352(20.7 \%)$ were resistant to rifampicin (RIF) and 19190 (21.7\%) showed resistance to isoniazid (INH). The proportion of rifampicin resistant cases that were mono-resistant increased from $15.3 \%$ in 2011 to $21.4 \%$ in 2014 while INH mono-resistance (IMR) showed a range between $13.8 \%$ and $21.1 \%$. The multidrug-resistant tuberculosis (MDR-TB) rates increased from $18.8 \%$ to $23.9 \%$ and the proportion of MDR-TB cases that had extensively drug-resistant tuberculosis remained between $10.2 \%$ and $11.1 \%$. Most drug resistance was found in females between the ages of 15 and 44 years and the northern districts bordering high MDR-TB regions had the highest MDR-TB rates.

Conclusion Our findings show increasing RIF monoresistance (RMR) and a substantial amount of IMR. This highlights a need for an initial test that detects resistance to both these drugs so as to avoid using RIF monotherapy during continuous phase of treatment in patients with IMR. Furthermore, addition of INH will benefit patients with RMR. Although DR-TB is widespread, HIV and migration influence its distribution; therefore, TB control strategies should include interventions that target these aspects.

\section{BACKGROUND}

The WHO has declared multidrug-resistant tuberculosis (MDR-TB) a global crisis. MDR-TB is defined as resistance to isoniazid
Strengths and limitations of this study

- The study was performed in the country with one of the highest tuberculosis (TB) incidence rate and a largest HIV epidemic in the world.

- The analysed data involve a period of major shift in TB diagnostic algorithm.

- The patient level Xpert MTB/RIF data were not available in order to compare with the TB culture results.

- The absence of unique patient identifiers also affects the accuracy of the data as the removal of duplicates was imperfect.

(INH) and rifampicin (RIF). Despite the decline in the global incidence rates of tuberculosis (TB), drug-resistant (DR) TB cases are on the rise with 558000 estimated incident cases of MDR plus rifampicin-resistant (RR) TB and more than 230000 deaths in $2017 .{ }^{1}$ South Africa has one of the highest incidence of TB in the world which WHO estimated to be 567 per 100000 in 2017. ${ }^{1}$ In 2017 alone, South Africa had an estimated number of 14000 RR cases, the second highest number in Africa after Nigeria which has more than three times the South African population. ${ }^{1}$ Moreover, the first reported outbreak of extensively drug-resistant tuberculosis (XDRTB) (defined as MDR-TB plus resistance to any second line injectable and a fluoroquinolone) which caused global concern in 2005 was from the province of KwaZulu-Natal $(\mathrm{KZN})$ in South Africa. ${ }^{2}$ While the incidence of TB in KZN is proportional to other provinces in the country, it remains the highest drug-resistant TB (DR-TB) burdened province with almost one-third of the country's cases of DR-TB. ${ }^{3}$

Compounding the problem of TB in South Africa is the high rate of co-infection with 
HIV (about 60\%). ${ }^{1}$ While it is well known that HIV is associated with smear negative TB, smear microscopy was traditionally used in the initial diagnosis of TB because of its quick time to results and low cost. ${ }^{4}$ On the other hand, conventional TB culture is much more sensitive than smear microscopy, but its high cost, complexity and long delays in getting the results made it impractical for routine diagnosis of TB. Therefore, when the WHO endorsed the Xpert MTB/RIF (Cepheid GeneXpert, Sunnyvale, California, USA) in 2010, it was subsequently introduced in South Africa in 2011. The Xpert MTB/RIF is an automated nucleic acid amplification test that offers better detection of TB compared with smear microscopy with an added advantage of the ability to detect RIF resistance in less than 2 hours in clinical specimens. ${ }^{56}$

The implementation of Xpert in South Africa completely changed the testing algorithm for the diagnosis of TB. ${ }^{7}$ Xpert replaced smear microscopy in the initial diagnosis of TB and all patients that do not demonstrate RIF resistance are assumed to have drug susceptible TB and therefore initiated on standard first line TB therapy. Thus, Xpert rifampicin susceptible cases do not get a culture, so INH mono-resistance (IMR) is not routinely investigated. TB culture and drug susceptibility testing (DST) is only indicated for patients that demonstrate RIF resistance on the Xpert, paucibacillary TB cases missed by Xpert (HIV infected, children and extra-pulmonary TB) and patients that fail TB treatment.

Despite the recent changes in the diagnosis and management of $\mathrm{TB}$, there are no studies that have assessed their impact on culture confirmed TB. The level of IMR that is not routinely investigated with current diagnostic methods is unknown, but instead these patients are getting RIF monotherapy during the continuous phase of their first line TB therapy which could potentially fuel drug resistance. On the other hand, patients with RIF mono-resistance (RMR) can benefit from the addition of INH in their treatment. We therefore undertook this study to evaluate the amount of RMR and IMR, so as to ensure optimal and appropriate diagnostic algorithms. We also describe the drug resistance patterns and distribution among different age groups, genders and districts in KZN, South Africa. Understanding the patterns and distributions of DR-TB will inform targeted intervention in TB control in this high TB endemic region.

\section{METHODS}

\section{Study design}

The study is a retrospective observational study using laboratory data from 2011 till 2014.

\section{Study setting}

The KZN province is one of nine provinces in South Africa and its population of just over 10 million ranks second in the country. There are 77 public health hospitals (including 8 MDR-TB initiation sites) within 11 health districts. Provincial Mycobacterium tuberculosis (MTB) culture and DST are performed in one central academic laboratory.

\section{Laboratory procedures}

MTB isolation from clinical samples was routinely done using the automated BACTEC mycobacteria growth indication tubes (MGIT) 960 system (BACTEC MGIT Becton Dickinson, USA). Indirect line probe assay (LPA) (GenoType MTBDRplus assay, Hain Lifescience, Nehren, Germany) was performed on all positive MGIT cultures using standard methods. Thereafter, additional DST for INH, RIF, ofloxacin, streptomycin, kanamycin was performed for all $\mathrm{TB}$ culture positive cases using $1 \%$ agar proportion method on Middlebrook $7 \mathrm{H} 10$.

\section{Patient and public involvement}

The data used for this study were the routine TB diagnostic data, therefore there was no direct patient and public involvement.

\section{Data collection and analysis}

The TB culture and DST data were collected from the National Health Laboratory Service laboratory information system (LIS) which contains all electronic laboratory results. In the absence of a unique identifier, duplicates were removed using MRN number (number given by the laboratory to specimens from the same patient) and demographic data (name, surname and date of birth). The results were stratified according to the health districts, age and gender. For the analysis of age, cases without recorded age or date of birth were excluded.

Data were described using frequencies and proportions. Continuous data were described using means, SD and $95 \%$ CI. Categorical outcomes were tested using the $\chi^{2}$ test. Data were analysed using Stata V.14.

\section{RESULTS}

Between 2011 and 2014, a total of 951209 specimens were cultured for MTB in KZN (figure 1). The total number of specimens for which culture was requested, decreased annually with the average percentage difference (decline) of $-14.2 \%$ ((95\% CI $-42.3 \%$ to $13.9 \%)$ and (SD $11.3 \%)$ ) per year. Similarly, the MTB positivity rate decreased by $6.0 \%$ (from $15.6 \%$ to $9.6 \%$ ) (figure 2). After removing duplicates, there were 36 644, 30 208, 22568 and 14672 culture confirmed cases of TB in 2011, 2012, 2013 and 2014 , respectively. The average percentage decline in total positive TB cases was $27.1 \%$ ( (95\% CI $3.5 \%$ to $50.7 \%)$ and (SD 9.5\%)) per year.

About $85 \%$ (88 559) cases of culture positive TB had an LPA done to test for drug susceptibility against RIF and INH (table 1). Of these, $19190(21.7 \%)$ were resistant to INH and 18352 (20.7\%) were resistant to RIF. There were 953 RMR cases in 2011, 767 in 2012, 676 in 2013 and 667 in 2014. RMR refers to the proportion RIF resistant cases that are susceptible to INH. The RMR increased from $15.3 \%$ in 2011 to $21.4 \%$ in 2014 . Over the same 4-year 


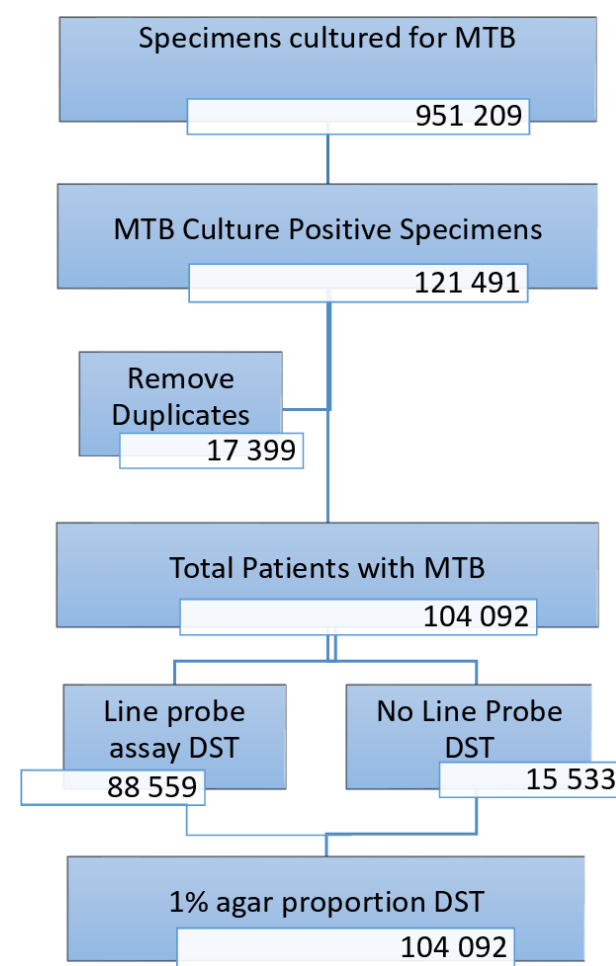

Figure 1 Flow diagram showing the number of specimens received and the laboratory procedures performed at the TB culture laboratory. TB, tuberculosis.

period, there were 3396 (17.7\%) IMR cases. IMR refers to the proportion INH resistant cases that are susceptible to RIF.

A steady decline of both MDR-TB and XDR-TB cases was noted, with an overall decline of $49.2 \%$ (from 6901 in 2011 to 3506 in 2014) and 44.9\% (from 706 in 2011 to 389 in 2014), respectively (table 2). The proportion of TB cases that had MDR-TB ranged from $18.8 \%$ in 2011 to $23.9 \%$ in 2014 , with an overall average of $21 \%$. The

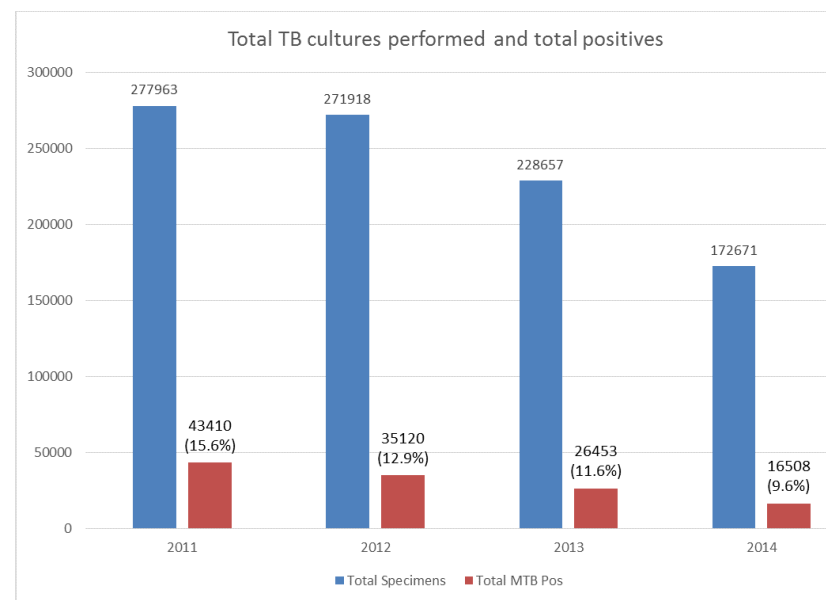

Figure 2 TB culture specimens processed between 2011 and 2014. The figure shows the total number of specimens received and the total number (and percentage) thereof that were positive. DST, drug susceptibility testing; MTB, Mycobacterium tuberculosis; TB, tuberculosis. overall rate of XDR-TB among MDR-TB cases was $11 \%$ (2336 XDR-TB cases out of 21221 MDR-TB).

The majority of the TB cases were males; however, females constituted highest prevalence of the DR-TB across all age groups (figures 3 and 4). The number of MDR-TB cases was higher among females than males until the age of 34 , thereafter males had a higher number than females. Similarly, for XDR-TB, females constituted the most number of XDR-TB. More than $60 \%$ of both MDR-TB and XDR-TB cases were patients between the ages of 25 and 44 years. It was observed that children less than 5 years of age showed the lowest rates of MDR-TB while that of XDR-TB was lowest between the ages of 6 and 14 years.

Over the 4-year period, eThekwini district had the highest number of TB cases with $47.5 \%$ of all cases in KZN coming from this district (table 2). However, the districts with the highest yearly proportion of MDR-TB cases each year were Umkhanyakude ( (mean 33.2\%, SD $2.3 \%)$, (95\% CI $29.5 \%$ to $36.9 \%)$ ); followed by Zululand ((mean $28.1 \%$, SD 2.3$)$, (95\% CI $24.4 \%$ to $31.8 \%)$ ) and Harry Gwala ((mean $26.2 \%$, SD 9.4), (95\% CI $11.2 \%$ to $41.2 \%))$. The yearly proportion of MDR-TB cases that had XDR-TB were highest at Umzinyathi ((mean $36.4 \%$, SD $5.8 \%)$, (95\% CI $27.2 \%$ to $45.6 \%)$ ) followed by eThekwini ((mean $13.7 \%$, SD $1.1 \%),(95 \%$ CI $11.9 \%$ to $15.5 \%)$ ) and Uthukela ((mean $13.2 \%$, SD $5.7 \%)$, (95\% CI $4.1 \%$ to $22.3 \%)$ ) districts. Umkhanyakude district had the lowest proportion of XDR-TB with a yearly mean of $3.4 \%$ ( $(\mathrm{SD}$ $2.4 \%)$, (95\% CI $-0.4 \%$ to $7.2 \%)$ ) over the study period (figure 5).

\section{DISCUSSION}

In this study, we observe a decline in the number of samples processed for MTB culture and culture positivity rate which coincided, with the roll out of the Xpert. This is in keeping with the Xpert roll out which started in March 2011 and was completed in September 2013 when all health facilities in the provinces were using the Xpert for TB diagnosis. According to the South African guidelines, MTB culture is not recommended for Xpert rifampicin susceptible patients, which constitutes the majority of patients infected with $\mathrm{TB}$, hence the decline in the number of MTB cultures from 277963 in 2011 to 172671 in 2014. Nevertheless, the sheer volumes of MTB cultures are still enormous which reflects the overwhelming burden of DR-TB in this region. Prior to Xpert introduction, DST for MTB was only performed on patients that were considered to be at risk of DR-TB, but the use of Xpert for initial diagnosis of TB enables screening for RIF resistance in all patients. The revised indications for culture selects for cases that are more likely to have DR (Xpert rifampicin resistant TB) and paucibacillary TB (extra-pulmonary TB and HIV positive Xpert negative), which explains the high rates of DR-TB and declining culture positivity rate observed in this study.

RIF is always used in combination with other drugs in the treatment of TB in South Africa. In addition, spontaneously 
Table 1 LPA results between 2011 and 2014: RIF and INH mono-resistance

\begin{tabular}{|c|c|c|c|c|}
\hline & 2011 & 2012 & 2013 & 2014 \\
\hline Total culture positives & 36644 & 30208 & 22568 & 14672 \\
\hline Total cases LPA & 31368 & 26513 & 18399 & 12279 \\
\hline LPA any INH resistance & 6430 & 5548 & 4167 & 3045 \\
\hline LPA any INH resistance (\%) & 20.5 & 20.9 & 22.7 & 24.8 \\
\hline LPA INH MR (\% of all INH resistant) & 13.8 & 21.0 & 21.1 & 17.1 \\
\hline LPA any RIF resistance & 6293 & 5013 & 3912 & 3134 \\
\hline LPA any RIF resistance (\%) & 20.1 & 18.9 & 21.3 & 25.5 \\
\hline LPA RIF MR & 953 & 767 & 676 & 667 \\
\hline LPA RIF MR (\% of all RIF resistant) & 15.1 & 15.3 & 17.3 & 21.3 \\
\hline
\end{tabular}

$\mathrm{INH}$, isoniazid; LPA, line probe assay; MR, mono-resistance; RIF, rifampicin.

occurring mutations are rare compared with other TB drugs. ${ }^{8}$ Consequently, the development of RMR is expected to be uncommon. The finding of increasing mono-resistance in this context is therefore concerning. In a previous study done by Coovadia $e t a l$, at the same laboratory, RMR was $8.8 \%$ during the years $2007-2009 .{ }^{9}$ Similarly, Mukinda et al reported increasing RMR in the Western Cape province of South Africa. ${ }^{10}$ These findings highlight the importance of testing for INH resistance in all patients with Xpert rifampicin resistance. This positively impacts patient management further as patients with confirmed RMR could benefit by using INH in their treatment regimen.

The development of RIF resistance has serious effects on the treatment of TB. Patients have to be treated with more expensive and more toxic drugs for a longer duration. Studies have been conducted in order to elucidate the causes of RMR with the majority reporting an association between HIV and RMR. ${ }^{11}{ }^{12}$ Factors contributing to this association include decreased drug bioavailability, and drug-drug interactions which lead to decreased RIF serum levels. ${ }^{13}$ Furthermore, advanced immunosuppression increases susceptibility to infection and permits proliferation of TB which favours transmission. ${ }^{14}$ Given the high rate of TB/HIV co-infection in our setting, it is possible that HIV may be contributing to the increasing rate of RMR. Whether using a higher dose of RIF proves to be beneficial in co-infected patients remains under investigation.

RIF and INH are core drugs that form the backbone for first line short course therapy for the treatment of drug susceptible TB. Given the high burden of disease in this region coupled with the use of Xpert as a screening tool for DR-TB, mono-resistance to INH may inevitably be overlooked. According to the national TB algorithm, a diagnosis of IMR TB is only made using TB culture and DST following a negative Xpert result or treatment failure. The use of standard first line TB therapy in patients with undetected INH resistance equates to using RIF monotherapy during the continuation phase. This may subsequently lead to the development of MDR-TB. This was described in a study done by Jacobson et al where treatment of patients with IMR using standard first line therapy was associated with poor outcomes and progression to MDR-TB. ${ }^{15}$ Several studies have reported previous TB therapy as a risk factor for IMR. ${ }^{16}{ }^{17}$ Identifying risk factors for IMR could help to select patients who may require TB culture and DST in order to exclude INH resistance.

There was an overall decline in the numbers of MDR (from 6901 in 2011 to 3506 in 2014) and XDR-TB (from 706 in 2011 to 389 in 2014) cases identified using culture. This was in contrast to the increasing number of MDR/RR-TB cases following the introduction of the Xpert in South Africa during this time. ${ }^{18-20}$ Perhaps a plausible explanation is that, contrary to the national guidelines, a significant number of patients with Xpert rifampicin resistant TB did not get a subsequent MTB culture for confirmation. This was supported by the 2016 WHO TB report, which reported the percentage of MDR-TB among MDR/RR-TB as $62 \%$ in South Africa. This suggests that a substantial number of Xpert rifampicin resistant TB cases are not confirmed by culture because this discrepancy cannot be explained by RMR cases. ${ }^{1}$ Another possible reason may be due to patients that are lost to follow-up. In KZN province, the specimen for TB culture is only collected when the patient comes back for Xpert results. Consequently, if patients did not return for the results, then specimens for TB culture would not have been collected. According to the WHO, only $41 \%$ of notified MDR/RR-TB cases from South Africa were enrolled for MDR-TB treatment in $2013 .^{18}$ Although this figure improved to $62 \%$ in $2014,{ }^{20}$ the gap remains substantial especially given the considerable improvement in rapidity of diagnosing DR-TB with Xpert. Therefore, this decline in culture confirmed that DR-TB indicates a change in the testing method used to diagnose TB rather than a successful TB control programme, which led to underestimation of MDR-TB and XDR-TB cases in this study. The proportion of MDR-TB cases that have XDR-TB remained constant at about $11 \%$ which is comparable to the global trends at that time. ${ }^{18-20}$ 


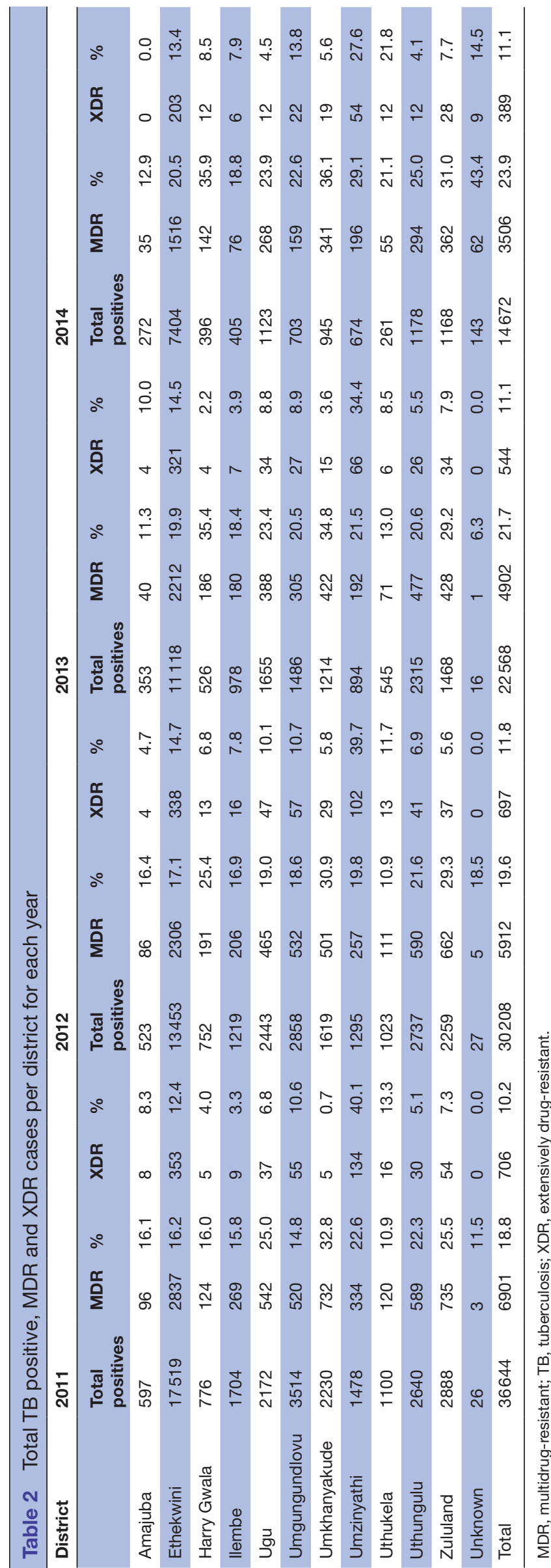

Our study found higher rates of DR-TB in women compared with men which supports findings from other studies showing higher proportions of DR-TB in women. ${ }^{21} 22$ Even though reasons behind the higher DR-TB predisposition among women are unknown, HIV could be a contributing factor. The majority of DR-TB cases were found between the ages of 15 and 44 years, which is the same age group that is known to have the highest HIV prevalence. ${ }^{2}$ It is well recognised that HIV is a major risk factor for development of TB and highly active antiretroviral therapy (HAART) reduces its incidence. ${ }^{23-25}$ Indeed, Nanoo et $a l$ showed an inverse relationship between antiretroviral therapy coverage and the incidence of microbiologically confirmed TB in South Africa, with the greatest decline demonstrated in the $25-44$ year age group. ${ }^{26}$

The diagnosis of DR-TB in children is generally difficult due to their inability to expectorate and the paucibacillary nature of childhood TB. Consequently, DR data are limited, but since TB in children is largely as a result of primary transmission from adults, the proportion of DR-TB is reported to be similar to that of adults. ${ }^{27}{ }^{28}$ Herein, we observed lower rates of DR-TB particularly MDR-TB in children less than 5 years compared with adults which could be a reflection of the under diagnosis of DR-TB in this age group. Although these rates are lower in children, they are still unacceptable as they reflect transmission of untreated adult TB.

Similar to the overall burden of TB in South Africa, DR-TB is also concentrated in urban areas of KZN with eThekwini district harbouring most of the cases due to high population density. However, the rate of MDR-TB cases was highest among the northern districts of the province of Umkhanyakude and Zululand. These are rural districts which share borders with Mpumalanga province, Swaziland and Mozambique, thus migration may influence resistance patterns. Mpumalanga province is known to have the highest DR-TB rate in the country while Swaziland has the highest MDR-TB prevalence in Africa. ${ }^{29}{ }^{30}$ In 2007, Wallengren $e t$ al reported Umzinyathi and Umkhanyakude as the districts with the highest MDR-TB rates. ${ }^{31}$ The intervention given to the Umzinyathi district following the outbreak of XDR-TB in 2005 (intensive case finding, early diagnosis and initiation treatment for $\mathrm{TB}$, early diagnosis and treatment of HIV, TB infection control and intergration of TB and HIV care) may be responsible for these decreasing rates. ${ }^{32}$ Despite declining XDR-TB rates at the Umzinyathi district (where the XDR-TB outbreak was identified in 2005), it still remains the district with the highest XDR-TB rates at about three times higher than the rest of KZN. ${ }^{33}$

\section{Limitations}

Our study is limited by the retrospective design; the accuracy of the data is dependent on available information on the LIS. The absence of unique patient identifiers also affects the accuracy of the data as the removal of duplicates is imperfect. Although duplicates were removed, we could not differentiate between new and known MDR-TB patients. The patient level Xpert data were not available in order to match with the TB culture results. Nevertheless, 


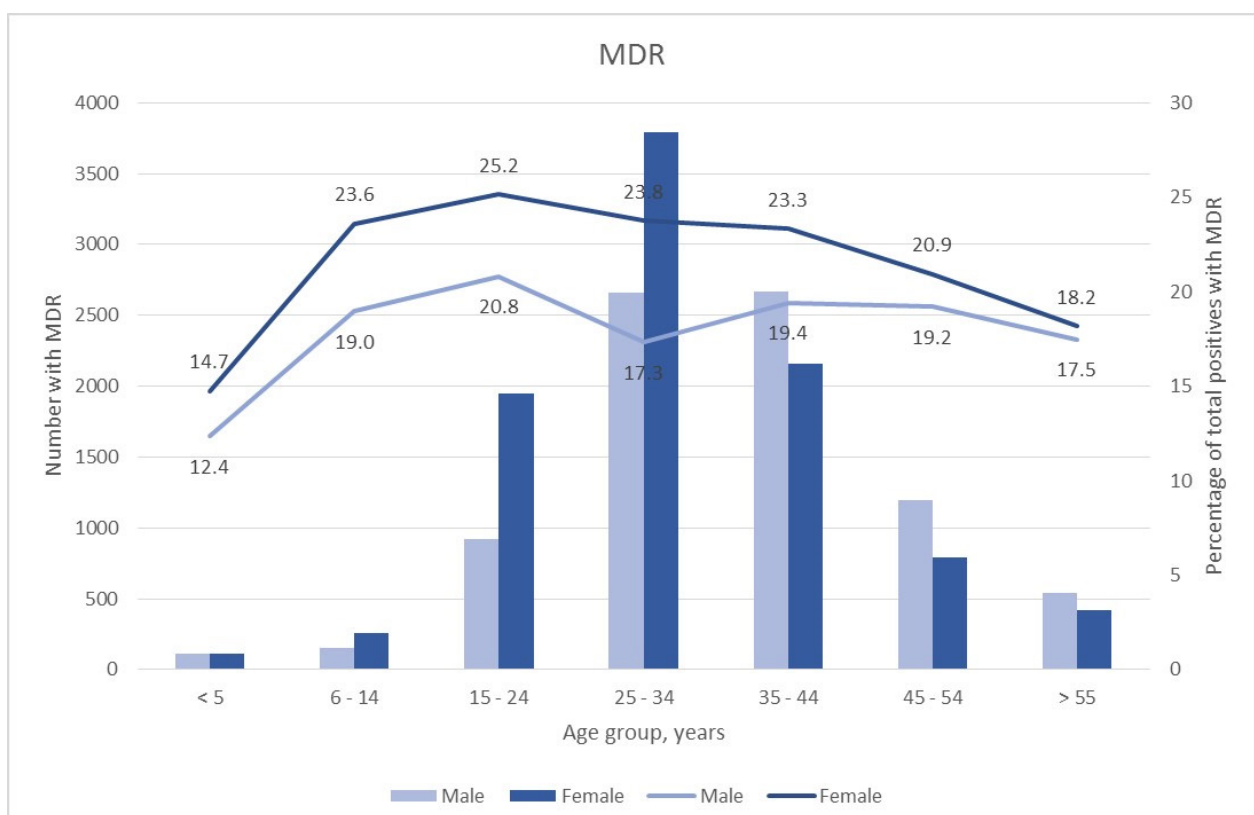

Figure 3 Distribution of MDR-TB cases and proportions by gender. The number of MDR-TB cases (represented by bars) by gender and the percentage (represented by lines) that is MDR-TB of total positive cases by gender. MDR-TB, multidrugresistant tuberculosis.

the high burden of DR-TB and the fact that all cultures are performed in one laboratory for the whole province provide an important insight to the distribution of TB in this region and may inform targeted intervention.

Although the data used for this study are relatively old, it represents a critical time of drastic changes in the diagnosis of DR-TB. There have been no subsequent changes in the TB diagnostic algorithm, therefore the findings highlighted in this study should still be relevant to the current setting. The data presented are not prevalence data as only data from MTB positive cultures were used. Thus, the results may be an underrepresentation as patients that were lost to follow-up and patients with contaminated/loss of viability cultures were excluded.

\section{CONCLUSIONS}

Our findings highlight the importance of DR-TB diagnostic algorithms that include both RIF and INH DST in the initial testing. Early detection of RMR will allow

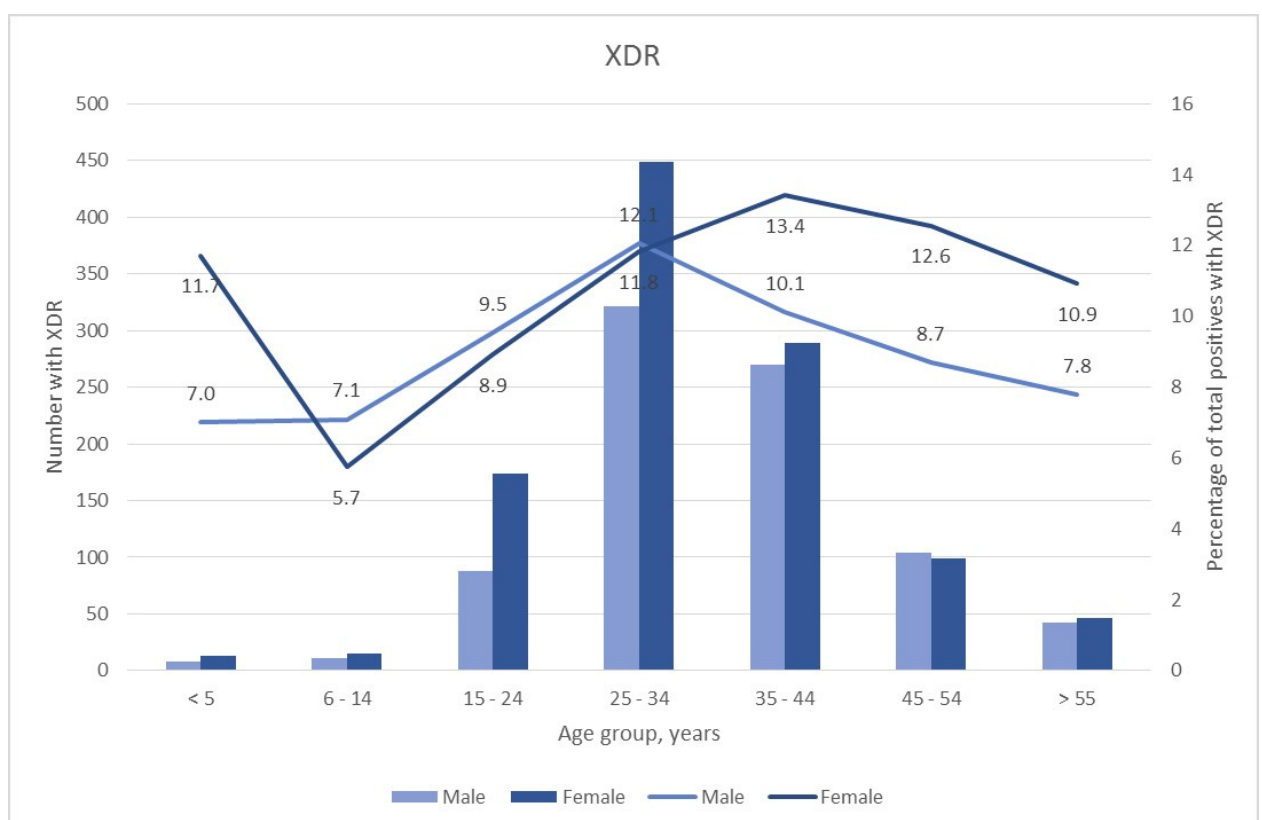

Figure 4 Distribution of XDR-TB cases and proportions by gender. The number of XDR-TB cases (represented by bars) by gender and the percentage (represented by lines) that is XDR-TB of total positive cases by gender. XDR-TB, extensively drugresistant tuberculosis. 

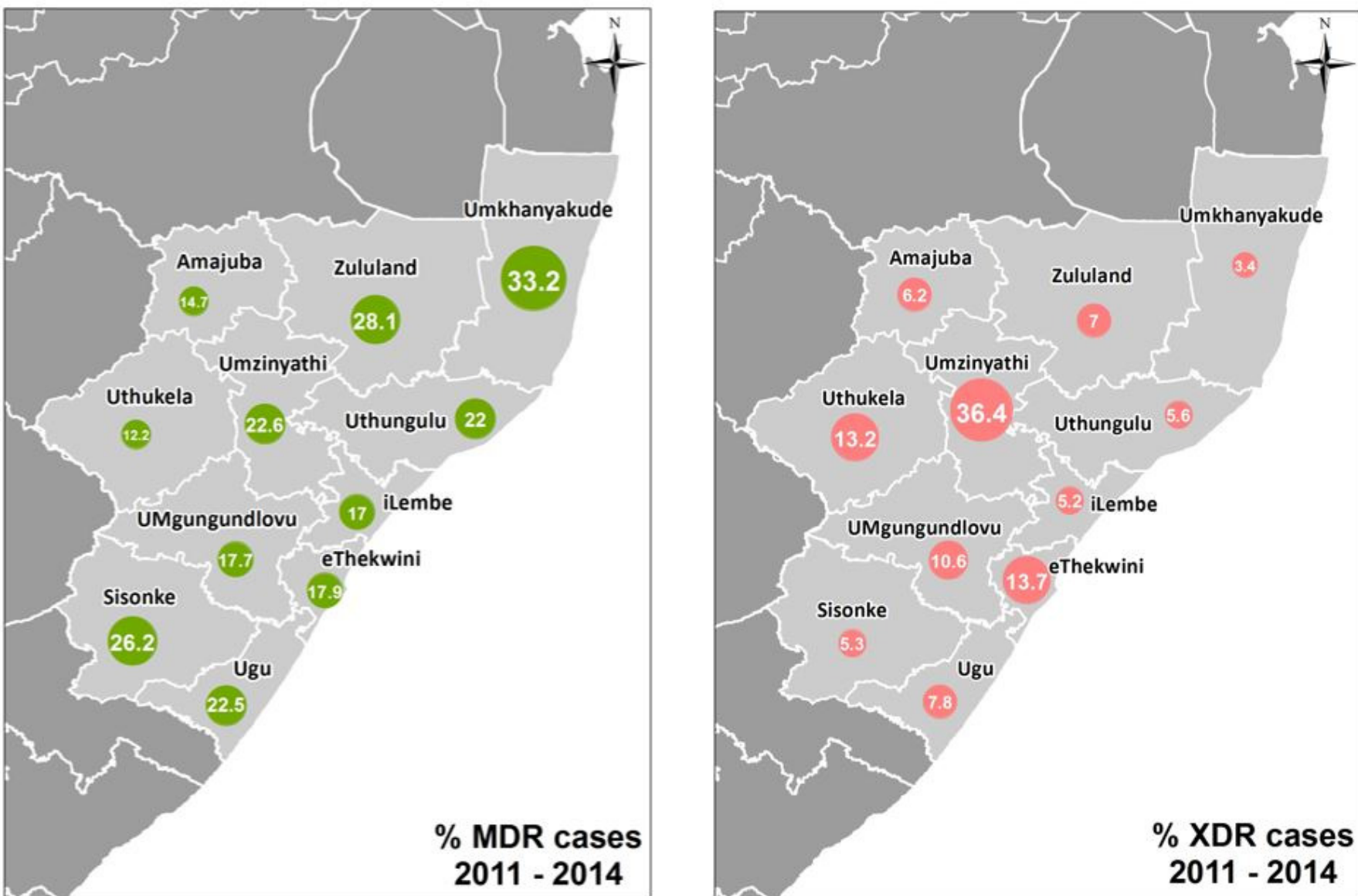

Figure 5 The first panel depicts the percentage of MDR-TB cases per district for the period 2011-2014. The percentage of MDR-TB cases among TB cases diagnosed by culture between 2011 and 2014. The size of the circle represents the percentage. The second panel depicts the percentage of XDR-TB cases per district for the period 2011-2014. The percentage of XDR-TB cases among MDR-TB cases diagnosed by culture between 2011 and 2014. The map was produced specifically for the purposes of this study, it is therefore not under copyright. MDR-TB, multidrug-resistant tuberculosis; XDR-TB, extensively drug-resistant tuberculosis.

addition of INH in the treatment regimen, while detection of IMR will prevent RIF monotherapy later on during the continuation phase of treatment which has been associated with development of RIF resistance. This will also allow us to have a clearer estimate of MDR-TB cases. HIV and migration play a significant role in the distribution of DR-TB in this region, therefore TB control measures that address these factors may have impact on DR-TB level.

Acknowledgements We thank the staff at the Inkosi Albert Luthuli Central Hospital TB laboratory for their dedication and hard work. We also thank Thandi Kapwata from the Environment and Health Research Unit within the South African Medical Research Council, for designing the provincial maps.

Contributors NRM contributed in the development of the concept, study design, data analysis and writing of the manuscript. YB performed data analysis and assisted with the writing of the manuscript. KL contributed in the interpretation of data and writing of the manuscript. KM supervised the development of the study concept, study design, data analysis and manuscript writing.

Funding The authors have not declared a specific grant for this research from any funding agency in the public, commercial or not-for-profit sectors.

Map disclaimer The depiction of boundaries on this map does not imply the expression of any opinion whatsoever on the part of BMJ (or any member of its group) concerning the legal status of any country, territory, jurisdiction or area or of its authorities. This map is provided without any warranty of any kind, either express or implied.
Competing interests None declared.

Patient consent for publication Not required.

Ethics approval The ethical approval to perform the retrospective analysis was obtained from the Biomedical Research Ethics Committee of the University of KwaZulu-Natal (REF: BE085/12). The data used for the study are routine data for management of TB patients; therefore, no individual patient consent was required.

Provenance and peer review Not commissioned; externally peer reviewed.

Data availability statement No data are available.

Open access This is an open access article distributed in accordance with the Creative Commons Attribution Non Commercial (CC BY-NC 4.0) license, which permits others to distribute, remix, adapt, build upon this work non-commercially, and license their derivative works on different terms, provided the original work is properly cited, appropriate credit is given, any changes made indicated, and the use is non-commercial. See: http://creativecommons.org/licenses/by-nc/4.0/.

\section{ORCID iD}

Nomonde Ritta Mvelase http://orcid.org/0000-0002-6037-9679

\section{REFERENCES}

1 World Health Organization. Global tuberculosis report, 2018. Available: https://www.who.int/tb/publications/global_report/ gtbr2018_main_text_28Feb2019.pdf?ua=1

2 Gandhi NR, Moll A, Sturm AW, et al. Extensively drug-resistant tuberculosis as a cause of death in patients co-infected with 
tuberculosis and HIV in a rural area of South Africa. The Lancet 2006;368:1575-80.

3 Ndjeka N. National department of health. multi-drug resistant tuberculosis: strategic overview on MDR-TB care in South Africa, 2014. Available: https://www.health-e.org.za/wp-content/uploads/ 2014/03/Strategic_overview_of_MDR_TB_RSA.pdf

4 Hassim S, Shaw PA, Sangweni P, et al. Detection of a substantial rate of Multidrug-Resistant tuberculosis in an HIV-Infected population in South Africa by active monitoring of sputum samples. Clin Infect Dis 2010;50:1053-9.

5 Helb D, Jones M, Story E, et al. Rapid Detection of Mycobacterium tuberculosis and Rifampicin Resistance by Use of On-Demand, NearPatient Technology. J Clin Microbiol 2010;48:229-37.

6 Boehme CC, Nabeta P, Hillemann D, et al. Rapid molecular detection of tuberculosis and rifampicin resistance. $N$ Engl J Med 2010;363:1005-15.

7 National Department of Health, South Africa. National tuberculosis management guidelines, 2014. Available: https://www.health-e.org za/wp-content/uploads/2014/06/NTCP_Adult_TB-Guidelines-27.5. 2014.pdf

8 McGrath M, Gey van Pittius NC, van Helden PD, et al. Mutation rate and the emergence of drug resistance in Mycobacterium tuberculosis. J Antimicrob Chemother 2014;69:292-302.

9 Coovadia YM, Mahomed S, Pillay M, et al. Rifampicin monoresistance in Mycobacterium tuberculosis in KwaZulu-Natal, South Africa: a significant phenomenon in a high prevalence TB-HIV region. PLoS One 2013;8:e77712.

10 Mukinda FK, Theron D, van der Spuy GD, et al. Rise in rifampicinmonoresistant tuberculosis in Western Cape, South Africa. int $j$ tuberc lung dis 2012;16:196-202.

11 Sharling L, Marks SM, Goodman M, et al. Rifampicin-Resistant tuberculosis in the United States, 1998-2014. Clinical Infectious Diseases 2019;123.

12 Villegas L, Otero L, Sterling TR, et al. Prevalence, risk factors, and treatment outcomes of isoniazid- and rifampicin- Mono-Resistant pulmonary tuberculosis in Lima, Peru. PLoS One 2016;11:e0152933.

13 Gurumurthy P, Ramachandran G, Hemanth Kumar AK, et al. Malabsorption of rifampicin and isoniazid in HIV-Infected patients with and without tuberculosis. Clin Infect Dis 2004;38:280-3.

14 Bifani P, Mathema B, Kurepina N, et al. The Evolution of Drug Resistance in Mycobacterium tuberculosis: From a Mono-RifampicinResistant Cluster into Increasingly Multidrug-Resistant Variants in an HIV-Seropositive Population. J Infect Dis 2008;198:90-4.

15 Jacobson KR, Theron D, Victor TC, et al. Treatment outcomes of isoniazid-resistant tuberculosis patients, Western Cape Province, South Africa. Clinical Infectious Diseases 2011;53:369-72.

16 Cattamanchi A, Dantes RB, Metcalfe JZ, et al. Clinical characteristics and treatment outcomes of patients with Isoniazid-Monoresistant tuberculosis. Clin Infect Dis 2009;48:179-85.

17 Fox L, Kramer MR, Haim I, et al. Comparison of isoniazid monoresistant tuberculosis with drug-susceptible tuberculosis and multidrug-resistant tuberculosis. Eur J Clin Microbiol Infect Dis 2011;30:863-7.

18 World Health Organization. Global tuberculosis report, 2013 Available: http://apps.who.int/iris/bitstream/10665/91355/1/ 9789241564656_eng.pdf

19 World Health Organization. Global tuberculosis report, 2014. Available: http://apps.who.int/iris/bitstream/10665/137094/1/ 9789241564809_eng.pdf

20 World Health Organization. Global tuberculosis report, 2015. Available: http://apps.who.int/iris/bitstream/10665/191102/1/ 9789241565059_eng.pdf

21 O'Donnell MR, Zelnick J, Werner L, et al. Extensively drug-resistant tuberculosis in women, KwaZulu-Natal, South Africa. Emerg Infect Dis 2011;17:1942-5.

22 Cox HS, Orozco JD, Male R, et al. Multidrug-Resistant tuberculosis in central Asia. Emerg Infect Dis 2004;10:865-72.

23 Sonnenberg P, Glynn JR, Fielding K, et al. How soon after infection with HIV does the risk of tuberculosis start to increase? A retrospective cohort study in South African gold miners. J Infect Dis 2005;191:150-8.

24 Badri M, Ehrlich R, Wood R, et al. Association between tuberculosis and HIV disease progression in a high tuberculosis prevalence area. Int J Tuberc Lung Dis 2001;5:225-32.

25 Badri M, Wilson D, Wood R, et al. Effect of highly active antiretroviral therapy on incidence of tuberculosis in South Africa: a cohort study. The Lancet 2002;359:2059-64.

26 Nanoo A, Izu A, Ismail NA, et al. Nationwide and regional incidence of microbiologically confirmed pulmonary tuberculosis in South Africa, 2004-12: a time series analysis. Lancet Infect Dis 2015;15:1066-76.

27 Schaaf HS, Marais BJ, Hesseling AC, et al. Surveillance of antituberculosis drug resistance among children from the Western Cape Province of South Africa-An upward trend. Am J Public Health 2009;99:1486-90.

28 Zignol M, Sismanidis C, Falzon D, et al. Multidrug-Resistant tuberculosis in children: evidence from global surveillance. Eur Respir J 2013;42:701-7.

29 Andrews JR, Shah NS, Gandhi N, et al. Multidrug-Resistant and extensively Drug-Resistant tuberculosis: implications for the HIV epidemic and antiretroviral therapy Rollout in South Africa. J Infect Dis 2007;196:S482-90.

30 Sanchez-Padilla E, Dlamini T, Ascorra A, et al. High prevalence of multidrug-resistant tuberculosis, Swaziland, 2009-2010. Emerg Infect Dis 2012;18:29-37.

31 Wallengren K, Scano F, Nunn P, et al. Drug-Resistant tuberculosis, KwaZulu-Natal, South Africa, 2001-2007. Emerg Infect Dis 2011;17:1913-6.

32 Bateman C. Tugela Ferry's extensively drug-resistant tuberculosis 10 years on. S Afr Med J 2015;105:517-20.

33 Lim JR, Gandhi NR, Mthiyane T, et al. Incidence and geographic distribution of extensively drug-resistant tuberculosis in KwaZuluNatal Province, South Africa. PLoS One 2015;10:e0132076. 